\title{
First physics with ATLAS
}

\section{Dirk ZERWAS, on behalf of the ATLAS collaboration*}

LAL, Orsay

E-mail: zerwaselal.in2p3.fr

The expected performance of ATLAS for first physics is summarized. In particular the use of first data such as $Z$ boson production and decay as well as low mass resonances for the calibration and alignment of the detector is discussed.

Physics at LHC 2008

29 September - October 4, 2008

Split, Croatia

\footnotetext{
${ }^{*}$ Speaker.
} 


\section{Introduction}

The ATLAS experiment is one of the two main multi-purpose experiments in preparation for the Large Hadron Collider (LHC), the $14 \mathrm{TeV}$ proton-proton collider at CERN. At this conference, the status of the ATLAS detector is summarized in [1] and the commissioning of the detector is discussed in [2]. The LHC era started on September 10, 2008 with the first beams circulating in both rings at an energy of $450 \mathrm{GeV}$ per beam and the successful recording of the first beam-spill events in ATLAS. The effective startup of the LHC at higher energy and with collisions is foreseen for 2009. The first physics results expected from collision data will in fact be the calibration of the detector and the measurement of its performance. The expected performance of the detector and the reconstruction algorithms will be summarized in the next section. In particular, the reconstruction and identification of electrons, muons, jet/ETmiss, taus, $b$-tagging and trigger will be discussed. A description of the ATLAS detector can be found in [3] (and references therein) and a summary of its performance in [4].

The electroweak measurements at the LHC and ATLAS are discussed in $[4,5,6]$ and for QCD and top quark physics a summary can be found in [4, 7]. Here only selected results for expected new physics in the auto-calibrated or large cross-section regime will be presented in section 3 . The results and conclusions will be summarized in the last section.

The basis of all studies presented in the following sections are the ATLAS Computing System Commissioning (CSC) studies [4]. A large scale production of full simulation and reconstruction of more than 25 million events has been performed. The simulations covered the full range from single particles to complex physics events. The most precise detector description available at that time was used. Imperfections such as mis-alignment and additional material were introduced voluntarily to get a realistic estimate of the behavior of the detector and its effect on the first analyses. The CSC program was also a test of the analysis model.

\section{Expected Performance}

The cross-sections for QCD jet production are several orders of magnitude larger than, for example, the production of the $Z$ boson decaying to electrons. A high jet rejection while maintaining high efficiency is therefore essential at the LHC. ATLAS has obtained an efficiency of $64 \%$ for a jet rejection of $10^{5}$.

The first source of electrons at the LHC will be the copious production of electrons with rather low transverse energy from $J / \psi / \Upsilon$ decays. A good signal to noise ratio can be obtained so that the low-mass di-lepton resonances can be clearly identified above the jet background.

A second source of electrons is the process $Z \rightarrow e e$. The ATLAS electromagnetic calorimeter has a uniformity of $0.5 \%$ in regions of $\Delta \eta \mathrm{x} \Delta \phi=0.2 \mathrm{x} 0.4$. These regions will be inter-calibrated using the well known mass of the $Z$ boson. The Monte Carlo studies show that a long range constant term of about $0.4 \%$ can be obtained with an integrated luminosity of $100 \mathrm{pb}^{-1}$. Thus the goal of a global constant term of $0.7 \%$ is well within reach.

A second use of the $Z$ boson is the determination of the electron reconstruction and identification efficiency from data only. One electron is well identified (tag) and the other electron is used to study the efficiency (probe). The results of the tag-and-probe method were compared to 

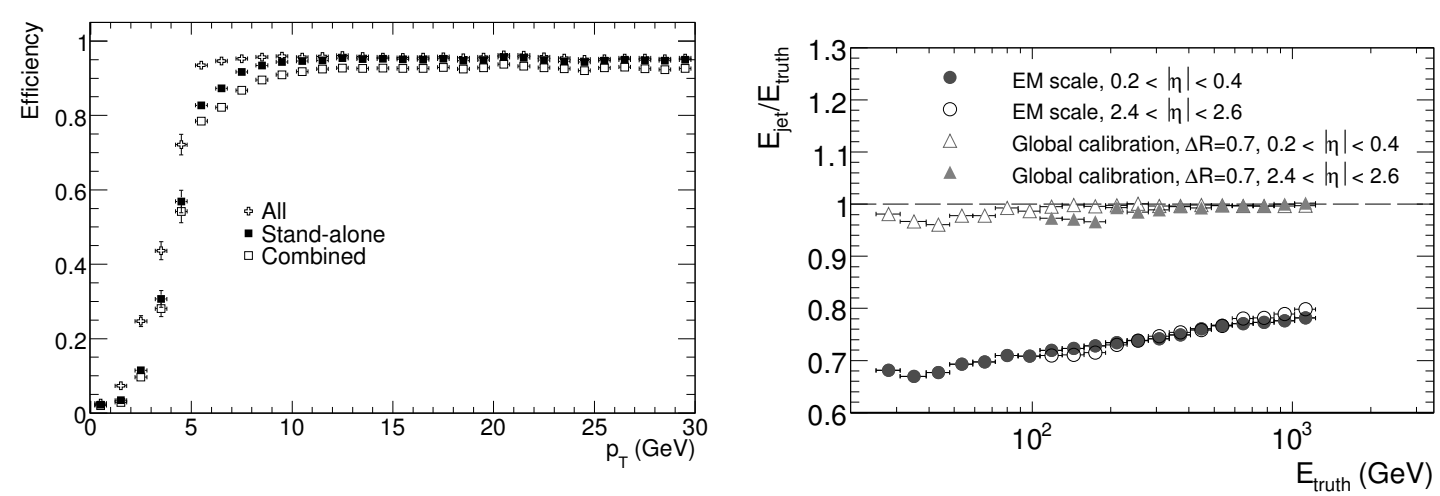

Figure 1: (Left) Efficiency for reconstructing muons as a function of the transverse momentum. The results are shown for muon spectrometer reconstruction, combined reconstruction and combined with the use of segment tags. (Right) Signal linearity for jets with $R_{\text {cone }}=0.7$, i.e., the ratio of reconstructed jet energy to the truth-jet energy, in two different regions of $|\eta|$ as a function of $\mathrm{E}_{\text {truth }}$ for jets at the electromagnetic energy scale and fully calibrated jets.

the efficiency determination from Monte Carlo truth. Good agreement between the two methods was found. With an integrated luminosity of $100 \mathrm{pb}^{-1}$ the efficiency can be determined with a statistical error of $\pm 0.1 \%$ and a systematic error of $\pm 1.5 \%$.

Muons are reconstructed in ATLAS using the muon spectrometer standalone and/or in combination with the inner detector. An excellent efficiency of more than $95 \%$ with a low fake rate is obtained for tracks with transverse momenta of more than $5 \mathrm{GeV}$ as shown in Figure 1 (Left) as well as good coverage in $\eta$. The combination of the muon spectrometer with the inner detector typically leads to a reduction of the expected fake rate by an order of magnitude.

The muon efficiency can be determined $\left(100 \mathrm{pb}^{-1}\right)$ with a statistical precision of $0.1 \%$ and a systematic error of $1 \%$ using the $Z$ boson production with the tag-and-probe technique. Additionally the muon energy scale, for muons with a transverse momentum of $50 \mathrm{GeV}$, using an integrated luminosity of only $50 \mathrm{pb}^{-1}$, will be determined with an accuracy of $\pm 0.5 \mathrm{GeV}$. The importance of the detector alignment can be illustrated by the following observation: while the central value of the $\mu \mu$ invariant mass distribution is not strongly affected by an initial misalignment of $1 \mathrm{~mm}$ and $1 \mathrm{mrad}$, the distribution is about three times broader than in the ideal case.

Jet reconstruction in ATLAS starts from reconstructed clusters calibrated in the local hadronic calibration scheme [4] or from towers. Jets are reconstructed using different types of algorithms, the most common being the cone and the kt algorithms. Jet energy scale corrections are then applied to correct for noise and pileup. As last stage, corrections for the underlying event are applied leading to refined jets.

The resulting jet energy scale is in agreement with the true (Monte Carlo) energy at the percent level as shown in Figure 1 (Right). The effect of the local calibration can be quantified by reconstructing jets at the electromagnetic energy scale: the offset from the true energy scale is of the order of $30 \%$. In-situ calibration will be carried out with two processes: photon plus jets and $Z$ boson plus jets. For transverse energies of less than $80 \mathrm{GeV}$ the statistical power of the first process is expected to be better than a percent for an integrated luminosity of only $10 \mathrm{pb}^{-1}$. Below 

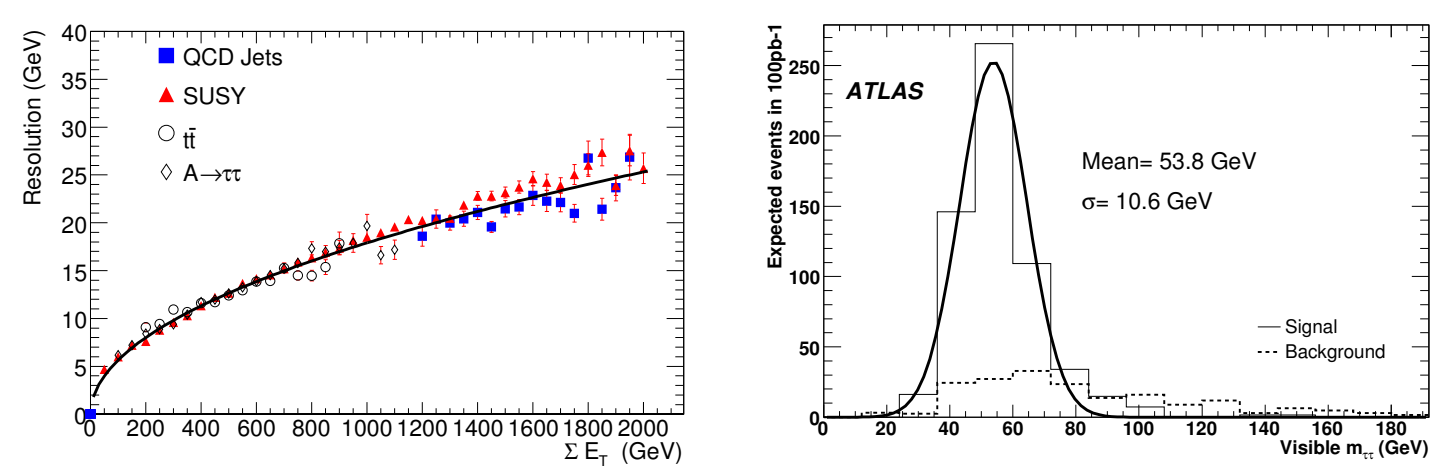

Figure 2: (Left) Resolution of the ETmiss vector after refined calibration as a function of the total transverse energy, measured in the calorimeters for different physics processes. The curve corresponds to $\sigma=0.57 \sqrt{\Sigma \mathrm{E}_{\mathrm{T}}}$. The points are for A masses from 150 to $800 \mathrm{GeV}$. The QCD jets are di-jet events generated with $560<p T<1120 \mathrm{GeV}$. (Right) The reconstructed visible mass of the $\left(\ell \tau_{\text {had }}\right)$ pair for $Z \rightarrow \tau \tau$ decays (solid line) and QCD, $W \rightarrow \ell \nu, Z \rightarrow \ell \ell$ backgrounds (dashed line).

$80 \mathrm{GeV}$ the background is non-negligible. The $Z$ (to electrons) boson production with additional jets can be observed clearly above the background with an integrated luminosity of $200 \mathrm{pb}^{-1}$.

If the ETmiss reconstruction were based on the calorimeter cells calibrated at the electromagnetic scale, the linearity would be offset by about $30 \%$ over most of the transverse energy range. Refining the reconstruction with knowledge of muons, electrons and jets, allows one to improve the linearity to about $5 \%$. The ETmiss resolution is expected to be about $0.57 \% \cdot \sqrt{\Sigma E_{\mathrm{T}}}$ as shown in Figure 2 (Left). A first check of the performance is the $\mathrm{Z}$ boson to $\tau \tau$ process. Here the scale of ETmiss can be checked albeit for low ETmiss values to within $2 \mathrm{GeV}$.

Two approaches are used for the reconstruction of the hadronic decays of the $\tau$-lepton in ATLAS: one is calorimeter based; the other one is tracking based. Comparison of independent methods is particularly useful in the startup of an experiment to understand the detector and reconstruction performance. Typically efficiencies of the order of $30 \%$ can be obtained for a jet rejection of $10^{3}$.

First physics results of the performance of the $\tau$ reconstruction will come from the study of the production of the $W$ and $Z$ bosons. Requiring ETmiss greater than $60 \mathrm{GeV}$ is sufficient to obtain a clean sample of $\tau$ candidates from the $W$ boson decay. The invariant mass of the $Z$ boson is reconstructed from the visible $\tau$ decay products where one $\tau$ decays leptonically and the other one hadronically. The visible mass in this channel is expected to peak at about $54 \mathrm{GeV}$ (Figure 2 (Right)). This process will then be used to calibrate the hadronic $\tau$ decay.

An important aspect for physics analyses is the flavor tagging capability of ATLAS. The performance of $b$-tagging can be summarized as follows (for details: [4, 8]): For a working point at $60 \%$ efficiency the rejection of light jets using the simplest algorithm, the combination of the signed impact parameter significance of the selected tracks in the jet, is about 30. Soft-lepton tagging will most likely be commissioned later. Using the IP3D method, i.e. the longitudinal and transverse impact parameter significance, the rejection is increased by a factor two. The most powerful method is the reconstruction of the secondary vertex where a light jet rejection of 150 is expected. 

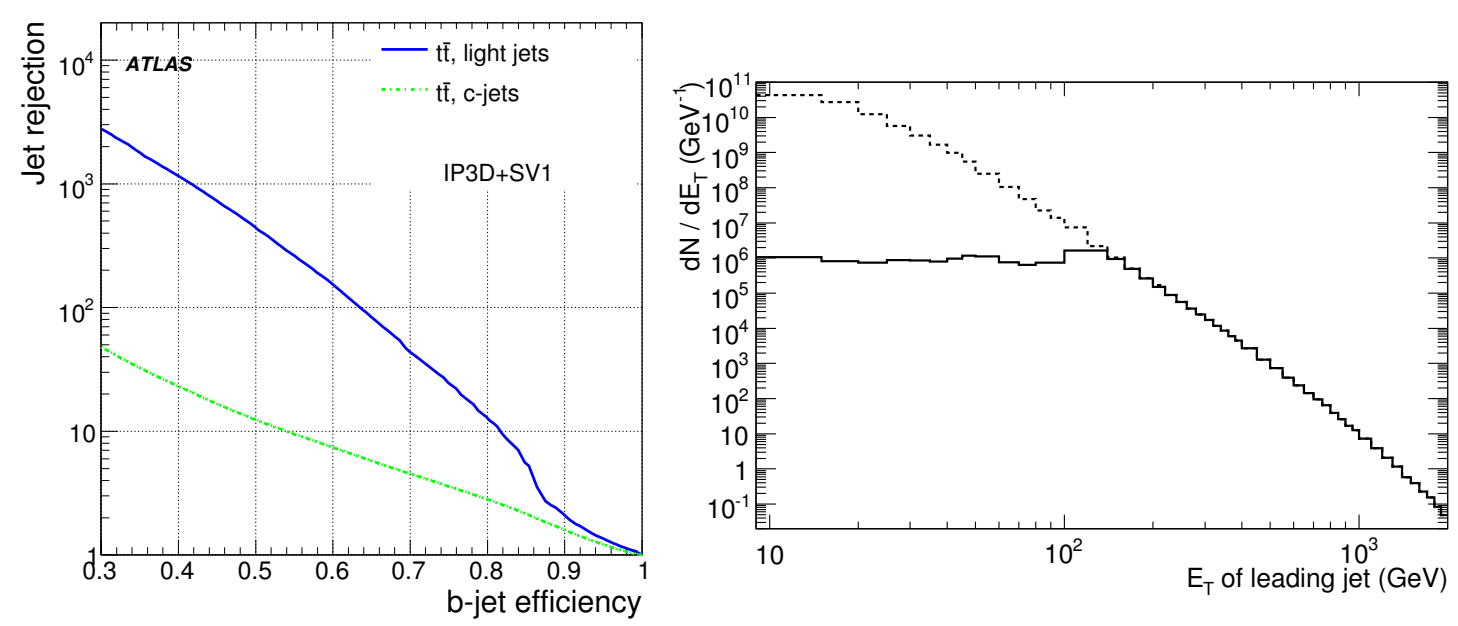

Figure 3: (Left) Jet rejection as function of $b$-jet efficiency in top samples. (Right) Expected differential spectrum for single jets as a function of the reconstructed ET of the leading jet. Solid line: after application of the L1 trigger thresholds and pre-scale factors, dashed line: expected distribution without any trigger requirements.

In situ calibration of $b$-tagging is based on top quark pair production. Comparing the $b$-tagging efficiency in situ (MC-Data) with Monte Carlo truth, good agreement has been found. A precision of $5-10 \%$ is estimated. Additionally, using muon plus jet events with low muon and jet energies is also foreseen. For this process, with $50 \mathrm{pb}^{-1}$ good agreement between MC-Data and MC-Truth was found for transverse energies of less than about $80 \mathrm{GeV}$.

Last, but certainly not least, the expected performance of the trigger has been estimated. Different trigger menus have been developed, e.g. for an instantaneous luminosity of $10^{31} \mathrm{~cm}^{-2} \mathrm{~s}^{-1}$ with relatively low thresholds in order to to able to accumulate large statistics of low mass resonances such as $J / \psi$ and $\Upsilon$. The total rates are within budget and the turn-on curves are reasonably sharp. It is interesting to note that, as shown in Figure 3 (Right), an integrated luminosity of $100 \mathrm{pb}^{-1}$ will allow the differential jet production to be studied over many orders of magnitude in ATLAS. The figure shows both the expected cross-section (dashed line) as well the rate after trigger (level-1, L1) and prescale requirements have been applied.

\section{First Physics}

Due to the large production cross-section for top quarks the LHC will be a top quark factory. A first analysis, assuming that the lepton identification efficiencies for electrons and muons are understood as well as jet reconstruction, will be the semi-leptonic channel without the use of $b$ tagging for a check of the production cross-section.

About 500 signal events are expected in the muon channel alone for an integrated luminosity of $100 \mathrm{pb}^{-1}$. Even without $b$-tagging, a clear signal will be visible above the background. The background can be determined from the data alone. A relative precision of $\Delta \sigma / \sigma= \pm 7 \%$ (stat) \pm $15 \%$ (syst) $\pm 3 \%$ (pdf) $\pm 5 \%$ (lumi) is expected to be achieved. The robustness of this result has 

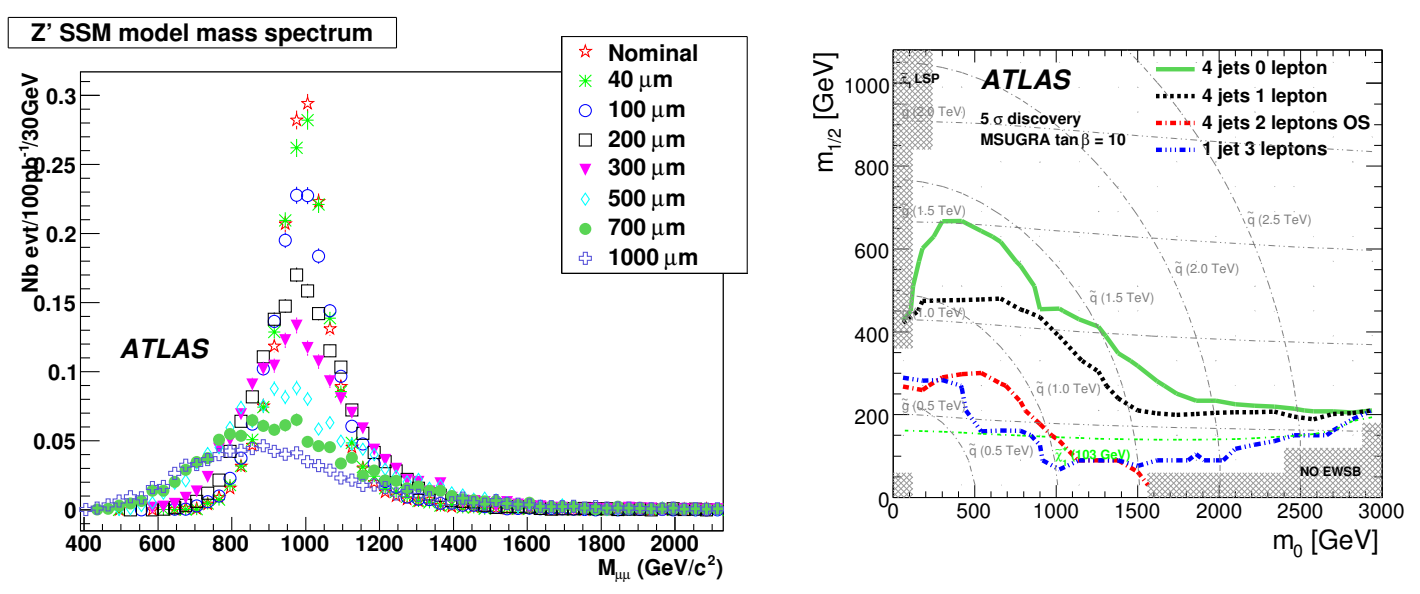

Figure 4: (Left) mass of the $Z^{\prime} \chi$ model for several misalignment scenarios. (Right) The discovery reach $(5 \sigma)$ for supersymmetry with $1 \mathrm{fb}^{-1}$ is shown for the lepton plus jets channel as black dashed line for $\tan \beta=10$.

been studied by increasing the QCD background cross-section by a factor two: the signal significance would be reduced by only about $30 \%$ and the signal would still be clearly visible.

In general at the LHC, a discovery will be made only once the Standard Model has been rediscovered and re-measured to ensure that the detector and reconstruction performance as well as the Standard Model are well understood. The exceptions are cases where the background for a new physics signal can be determined entirely from the data alone, without relying on the Monte Carlo modeling of the background. One such example is the search for extra gauge bosons such as a $Z^{\prime}$ or $W^{\prime}$ decaying into pairs of electrons and muons or electron/muon and large ETmiss. Such a study of course necessitates a good understanding of reconstruction and identification of electrons and muons as discussed in the previous section. Additionally, for the $W^{\prime}$, ETmiss must be understood.

ATLAS is sensitive to $Z^{\prime}$ boson masses of about $1.2-1.5 \mathrm{TeV}$ with an integrated luminosity of about $100 \mathrm{pb}^{-1}$. For the $W^{\prime}$ boson the sensitivity is even greater, reaching $W^{\prime}$ masses of about $2 \mathrm{TeV}$. The discovery ranges vary as function of the model parameters. As shown in the previous section for the reconstruction of $\mathrm{Z}$ boson decays to muon pairs, the $Z^{\prime}$ discovery in the muon channel will depend on the quality of the alignment. The initial misalignment of about $1 \mathrm{~mm}$ reduces significantly the number of signal events in a given mass bin as shown in Figure 4 (Left). The current limits, from the Tevatron and LEP, are of the order of $500 \mathrm{GeV}$ to $1 \mathrm{TeV}$ [9] making these channels primary discovery channels at the LHC with a relatively small amount of integrated luminosity.

The final states for the production of moderately heavy supersymmetric particles such as squarks and gluinos have large cross-sections and lead to final states with several large transverse momentum jets. At least two neutralinos escape undetected. This leads to large ETmiss, but also prevents a direct reconstruction of the (s)particle masses. In the rather long (cascade) decay chains, edges and thresholds can be observed by combining reconstructed jets and leptons.

An important inclusive variable, before analysing the details of decay chains, will be the effective mass. It is constructed by summing the jet transverse momenta and ETmiss, thus relying on 
a well understood detector. Requiring additionally at least one lepton will reduce the signal crosssection, but is also safer with respect to the estimation of the background which would be pure QCD without this requirement. A good separation of signal and background is obtained for high values of the effective mass. A sensitivity of squark and gluino masses of the order of $400 \mathrm{GeV}$ will be reached with an integrated luminosity of only $10 \mathrm{pb}^{-1}$. Such an analysis will be competitive with the current limit on squark and gluino masses from the Tevatron. With a larger integrated luminosity of $1 \mathrm{fb}^{-1}$ a sensitivity to masses of about $1 \mathrm{TeV}$ would be reached as shown in Figure 4 (Right).

\section{Conclusions}

The CSC studies have shown that ATLAS is well prepared for the effective start of the LHC in 2009. The expected performance of the reconstruction of electrons, muons, taus, jets, ETmiss, $b$-tagging as well as the trigger menu for initial startup is well understood. The first physics results expected from ATLAS will rely on low mass resonances as well as the $Z$ boson production and leptonic decays to determine the performance from data.

ATLAS is eagerly awaiting next year's data at $10 \mathrm{TeV}$ (or more) to find: the Standard Model, search for exotic new physics and supersymmetry or whatever nature has in store in the new energy regime.

\section{Acknowledgments}

The author would like to thank his colleagues in ATLAS for numerous discussions in the preparation of the talk and manuscript. It was a pleasure to attend the conference "Physics at LHC 2008 " in the beautiful town of Split. I would like to thank the organizers for the remarkable job.

\section{References}

[1] Peter Jenni, in proceedings of Physics at LHC 2008 POS ( $2008 \mathrm{LHC}$ ).

[2] Manuella Vincter, in proceedings of Physics at LHC 2008 POS (2008LHC).

[3] ATLAS Collaboration, The ATLAS Experiment at the CERN Large Hadron Collider, JINST 3 (2008) S08003.

[4] ATLAS Collaboration, Expected Performance of the ATLAS Experiment, Detector, Trigger and Physics, CERN-OPEN-2008-020, Geneva, 2008, to appear.

[5] Lucia di Ciaccio, in proceedings of Physics at LHC 2008 POS (2008LHC).

[6] Kristin Lohwasser, in proceedings of Physics at LHC 2008 PoS (2008LHC) .

[7] Frank-Peter Schilling, in proceedings of Physics at LHC 2008 POS (2008LHC).

[8] Laurent Vacavant, in proceedings of Physics at LHC 2008 PoS (2008LHC).

[9] C. Amsler et al. (Particle Data Group), Physics Letters B 667 (2008) 1. 